Author 1- Dr. Shruti Singh,

Post graduate, Department of Periodontology, Manipal College of dental Sciences, Manipal.

Author 2- Dr. Rupanjan Roy,

Post Graduate, Department of Periodontology, Manipal College of dental Sciences, Manipal. 


\begin{abstract}
Hyaluronic acid, known also as hyaluronan, is the most abundant glycosaminoglycan in the extracellular matrix. It is produced by most of the cells in the human body, possibly contributing to various fundamental biological processes. This prompts interest in its therapeutic value. Hyaluronic acid (HA) has been used for various treatments owing to its broad range of properties in medical and dental field specially in periodontology. The review gives an overview on the effects of HA on fibroblasts, bone, wound healing, periodontal regeneration, aphthous ulcer/stomatitis and stem cells. Outcomes of including Hyaluronic acid in non-surgical periodontal therapy, drug delivery system and surgical procedures such as root coverage, papilla regeneration, root conditioning, intrabony defects, guided tissue regeneration are rather optimistic. HA has shown astounding potential in treating periimplantitis. Newer frontiers have to be explored to comprehend the other promising properties of HA.
\end{abstract}

Keyword - Hyaluronic Acid, Periodontology. 


\section{POTENTIAL FOR HYALURONIC ACID IN PERIODONTAL RESEARCH}

\section{Introduction}

Hyaluronic acid, known also as hyaluronan, is the most abundant glycosaminoglycan in the extracellular matrix(1). This linear polysaccharide has multiple repeating disaccharide units $[(1 \rightarrow 3)-\beta-d G l c N A c-(1 \rightarrow 4)-\beta-d-G l c A-](2)$. It is estimated that almost 15 grams of hyaluronic acid are present in the body of a normal adult weighing $70 \mathrm{kgs}(3)$. It is produced by most of the cells in the human body, possibly contributing to various fundamental biological processes. This prompts interest in its therapeutic value(4).

Hyaluronic acid has been used for various treatments owing to its broad range of properties. It is widely used in medical fields such as orthopaedics, ophthalmology, dermatology, cardiology, radiology. It has shown promise as a delivery system for vaccines and other medicines. Moisture retention is one role of HA that has been exploited well resulting in the availability of several pharmacological preparations. It is an attractive therapeutic option even in the field of dentistry owing to its pseudo-elasticity, viscoelasticity, biocompatibility, anti-inflammatory and bacteriostatic nature(5). HA forms a crucial element in the extracellular matrix of the soft tissues such as gingiva and periodontal ligament, while it is present in lesser quantities in the mineralized periodontal tissues of cementum and alveolar bone(3). The range of applications for HA in periodontal research are manifold and are summarized in this review.

There is consensus that wound healing is accelerated with HA. It acts via modulating specific HA receptors to regulate inflammation, cellular migration and angiogenesis, which are the main phases of wound healing. Sudhakar et al 2020 stated that proteins that bind to HA cause activation of CD44 surface receptors which facilitate cell adhesion, migration and differentiation(6). 
A retrospective study on healthy adults with minor recurrent aphthous stomatitis using a barrierforming HA mouthwash (GUM® AftaClear® rinse) and topical gel (GUM® AftaClear® gel) showed reduction of pain, and lesion dimensions during treatment. Complete lesion closure was obtained in 60 and $56 \%$ of the cases, respectively(7).

According to a systematic review by Casale et al in 2017, patients receiving HA for treatment of aphthous ulcers experienced faster and prolonged relief of symptoms accompanied by a reduction in the number of ulcers and inflammatory signs. Reasons for this improvement include suppression of immune response by HA and its antioxidant effect. Being hygroscopic and viscoelastic, it promotes re-epithelialization and reduces scarring. Further, the frequency of ulcer recurrence is also reduced, which is related to the inability of viral and bacterial penetration through the HA layer(8).

Hence, the wound healing property may be extrapolated to vesiculobullous lesions commonly seen on the gingiva. The topical form application of $0.2 \%$ hyaluronic acid, Hydroxychloroquine sulphate is suggested to be effective in oral erosive Lichen planus(9). Attempts at periodontal regeneration have also derived from this wound healing property of HA.

\section{Effects on Stem cells}

Umemura et al, 2016 conducted a study to assess the differentiation of odontoblasts in DPSCs (Dental Pulp Stem Cells) with the application of 1\% HA (Nacalai Tesque Co., Kyoto, Japan). The flow cytometry analysis suggested that the use of HA can lead to an upsurge of the cells that are responsible for the expression of CD44 cells. It increases by about $10 \% 5$ minutes posttreatment which showed a reduction to $54 \%, 30$ minutes post-treatment. HA treatment for a week showed an enhancing effect on the alkaline phosphatase protein levels and therefore on DPSC mineralization. The mRNA levels of DSPP and DMP-1 (odontoblastic differentiation 
markers) increased 6.7 and 7.7 times respectively thereby increasing the protein levels as well without having any effect on the odontogenic differentiation markers. This action takes place due to HA's property of enhancing CD44 signalling(10).

Schmidt et al 2020 assessed the impact of different concentrations of Low Molecular Weight Hyaluronic Acid (LMW-HA) (800 Da, 1600Da, $15000 \mathrm{Da})$ on the proliferation activity, cell size, and phenotypic surface markers of DPSCs. The cell viability was seen to be greater than $94 \%$ in all the test groups and significantly lesser in the control group (80.2\%-97.2\%). The cell size did not show a significant difference between the groups. The proliferation was found to be statistically significant in all the test groups when compared to the control (C vs T1- 0.0015, C vs T2- 0.0143, C vs T3- 0.0864). The test groups showed significantly higher numbers of surface markers compared to the control group(5).

\section{Effects on fibroblasts}

Peck et al 2018, conducted an in vitro study on the effect of cross-linked HA (hyaDent BG, Bioscience $\mathrm{GmbH}$ ) and HA and L-PRF combination on fibroblast cell lines. Results established that HA is compatible with the fibroblasts and enhances cell viability and proliferation. Peak cell proliferation appeared to be on the $8^{\text {th }}$ day $(112.20 \% \pm 9.69)$ and the $7^{\text {th }}$ day $(100.88 \% \pm$ $96.15)$ in the combination group(11).

It is also believed that the viability of the periodontal fibroblasts increases with the use of HA as it boosts alkaline phosphatase levels. Kobayashi et al 2017 investigated the effects of noncross-linked and cross-linked HA (hyaDENT, BioScience GmbH, Dümmer, Germany and hyaDENT BG, BioScience GmbH) on human PDL fibres at different dilutions of 1:10, 1:100 and direct coating. Higher numbers of viable cells were seen in both the groups at 3 and 5 days. A significant increase in alkaline phosphatase and collagen was seen in the presence of the 
osteogenic differentiation medium in all the groups but more at 1:10 dilution of both non-crosslinked and cross-linked HA(12).

\section{Effects on bone}

HA was also shown to enhance the osteoblast production and differentiation leading to more mineralization. Positive effects on CD44 cells and their expressions also increase the rate of bone formation. New bone formation, angiogenesis and degradation of residual grafts are all favoured when the graft is placed along with HA.

Arpağ 2018 conducted a study on rabbits where HA was used along with xenograft ((Orthovisc $2 \mathrm{ml}$, Biomeks, Istanbul, Turkey) + (Integros BonePlus®, Integros, Adana, Turkey)) for filling bony defects. When compared with xenograft alone, the combination group showed better results than xenograft histologically (new bone formation- 26.90 vs 30.72; marrow space- 11.01 vs 14.49 ; residual graft- 63.59 vs 54.78 ). The combination of xenograft and HA showed lesser improvement than autograft(13).

Cirligeriu et al 2018 conducted an in vitro study on the interaction of HA (Hyadent BG, Bio Science, Dummer, Germany) with the chorioallantoic membrane in presence or absence of bone graft and/or saline. HA showed increased vascularisation in 1 day and suffusion bleeds within 2 days of administration. With bone, HA showed an interlaced and compact layout of lamella with enhanced angiogenesis on the seventh day. HA with saline and bone induced wheel spokes arrangement of blood vessels(14).

Hyaluronic acid may act as a biomaterial scaffold for other molecules, such as BMP-2 and PDGF-BB(15). Hyaluronic acid shares bone induction characteristics with osteogenic substances such as bone morphogenetic protein-2 and osteopontin. Hyaluronic acid accelerates the bone regeneration using chemotaxis, proliferation and successive differentiation of mesenchymal cells(16). 
Kim et al 2016 evaluated the histological effect of 1\% HA (Healon, Pharmacia \& Upjohn, Uppsala, Sweden) on endodontic and periodontic combination lesions in bagel dogs. The mineralization density of the bone in the test group was statistically higher as compared to that of the control group. In the test group, more reversal lines could be seen indicating a greater number of osteoblasts and secondary osteons(17).

\section{Non- surgical periodontal therapy}

HA can be used as an adjunct to SRP in gingivitis and chronic periodontitis patients. It has shown improved healing and periodontal indices including CAL improvement.

Madkour et al 2018 conducted an RCT to assess the effects of HA in chronic periodontitis patients (CAL $\geq 3 \mathrm{~mm}$ and $\mathrm{PPD} \geq 4 \mathrm{~mm}$ in at least 4 sites) with a medical history of controlled DM II (HbA1c- 6\%-7\%) and under medication. 1mL HA (Hyadent BG, BioScience GmbH, Ransbach-Baumbach, Germany) was applied subgingivally into the pockets, as an adjunct to SRP (scaling and root planing). The periodontal parameters were assessed at baseline, 6 and 12 weeks and compared to SRP alone. No significant difference was seen between the groups in Plaque index at both intervals. Gingival index (GI), PPD, CAL improvement was seen in both the groups but more with SRP+HA. Where statistically significant $\mathrm{p}$-value was $\leq 0.05$, GI at 6 weeks was 0.0485 , GI at 12 weeks was 0.0457 , PPD at 6 weeks was 0.0130 , PPD at 12 weeks was 0.0407, CAL at 6 weeks was 0.0477 and CAL at 12 weeks was $0.0232(18)$.

A systematic review by Casale et al 2016 reviewed three studies with gingivitis, thirteen studies with chronic periodontitis, seven studies with surgeries and three with oral ulcers where topical HA gel was used. Two different concentrations of HA gel $(0.2 \mathrm{~mL} / 0.5 \mathrm{~mL} / 1 \mathrm{~mL})$ were used in the studies- $0.2 \%$ (Gengigel) and $0.8 \%$ for different time periods. The studies showed that HA gel, when applied topically showed positive results in healing, reduction in inflammation, 
plaque index, gingival index, bleeding index, probing pocket depth and clinical attachment level. It has also shown an increase in the sulcular fluid flow rate [6]. Sudhakar et al 2020 observed that along with scaling and root planing, 0.2\% HA (topical-gel/spray) B.I.D 8 X 3-5 weeks has given positive results in chronic periodontitis(19).

\section{ANTIOXIDANT EFFECT}

Free radicals and oxidative stress have a major role to play in the development and progression of periodontal diseases. HA has been proven to have an antioxidant effect and therefore has a beneficial effect in clinical outcomes of non-surgical periodontal therapy when used as an $\operatorname{adjunct}(20)$.

Aydinyurt et al 2020 conducted a study to assess the antioxidant effects of HA. HA was used as an adjunct to SRP in two different forms - Gel (Gengigel, $0.2 \% \mathrm{HA}$, gingival gel, Ricefarma S.R.L, Milan, Italy) and Mouthwash (Gengigel, 0.2\% HA, Hydrogel, Ricefarma S.R.L, Milan, Italy). The first group received only Scaling and Root Planing (SRP). The second group received SRP along with HA gel application subgingivally for 30 seconds. The third group received SRP and HA mouthwash (subgingival irrigation) whereas the fourth group received SRP + HA gel +HA mouthwash. PI, GI, BOP, PD, GRN (Gingival Recession Height) were measured at baseline and after 4 weeks along with ADA (adenosine deaminase), CAT (catalase) and GSH (glutathione) levels in the GCF. ADA, CAT and GSH are considered to be important biomarkers for immunologic reactions as they are antioxidant enzymes. All the clinical improved in all the groups with no significant difference between the groups at 4 weeks. ADA levels decreased whereas CAT and GSH levels decreased significantly in all the groups. After 1 week a significant difference was found in the CAT and GSH levels between the groups (greater increase concerning group 2 and 4). Both HA gel and HA mouthwash showed antioxidant properties but the gel proved to be more effective. The results connotate that HA 
nullifies the effects of the reactive oxygen species and nascent oxygen load produced by the microbes and host immunity. The probable reason that HA gel group showed better results is that the gel stayed at the greater depth in the subgingival region for a relatively longer duration as compared to mouthwash. In group 4, the double action of the gel and mouthwash reduced the existing antioxidants and prevented further formation of them(3).

\section{Surgical periodontal treatment}

\section{RECESSION COVERAGE}

In recession coverage, HA use has shown improved CAL and an increased amount of keratinization. Pilloni et al 2019 used 1mL of Hyadent BG, BioScience GmbH, RansbachBaumbach, Germany, a combination of cross-linked and natural HA gel $(16 \mathrm{mg} / \mathrm{ml}$ and $2 \mathrm{mg} / \mathrm{ml}$ respectively) along with coronally advanced flaps (CAF) in Miller's class 1 recessions. After 7 days of surgery, patients in the test group experienced statistically significantly lower swelling and discomfort. 18 months post-operative evaluation revealed complete recession coverage and clinical attachment level gain of $3 \mathrm{~mm}$ in the test group whereas the control group (CAF only) showed improvement of $2 \mathrm{~mm}$. With HA, complete root coverage was seen in $80 \%$ of cases compared to $33 \%$ in the control group. Amount of keratinized tissue obtained after 18 months was similar in both the groups(21).

Patient discomfort post-surgery can be reduced with the help of HA. HA can also provide enhanced epithelialization and better colour match after surgery. It has also shown to reduce the inflammatory infiltrates thereby reducing the inflammation itself. Yıldirım et al 2017 conducted an RCT where HA was used for reducing post-surgical pain and improving palatal healing after procuring a free gingival graft (FGG). $0.2 \%$ and $0.8 \%$ HA gels (Gengigel, Ricerfarma SRL, Milano, Italy and Gengigel Prof, Ricerfarma SRL respectively) were applied 
on the donor site and covered with a periodontal pack on the day of surgery followed by another application 3 days after. The patient evaluation was done using a visual analogue scale (VAS), epithelialization and colour match at 3, 7, 14, 21, and 42 postoperative days. In both, the groups, no pain and burning sensation was experienced after 14 days whereas the control group showed slower improvement till 21 days. Complete epithelialization (CE) was seen in 21 days in both the groups (At 14 days, $8.3 \%$ and $50 \%$ epithelialization were seen in $0.2 \%$ and $0.8 \%$ groups respectively). The control group showed CE in 42 days. Better colour match was seen in the test groups in 21 days when compared to the control groups(22).

\section{PAPILLA REGENERATION}

Injectable HA in different concentrations has been successfully used for papillary regeneration and black triangles $(50 \%-100 \%)$. This is attributed to the property of HA to induce neovascularization.

Pi et al in 2017 conducted an RCT on female rats where either HA or Phosphate buffer solution (PBS) was injected in interdental papillae. HA group showed increased papillary volume (convex in shape) when compared with the PBS group (concave in shape). This in-vitro study stated that injecting HA fillers in the interdental papilla showed new micro vascularity in the connective tissue layer with the absence of inflammatory infiltrates in rats(23).

Bertl et al 2017 assessed the effect of HA in papilla regeneration in maxillary anterior implantsupported teeth. Injections were given in 3 different sites- mucosa above the mucogingival junction, just above the papilla in the attached gingiva, $2-3 \mathrm{~mm}$ apical to the tip of the papilla. The injection consisted of $1 \mathrm{ml}$ of HA gel (HYadent barrier gel, BioScience, Germany) which consisted of $16 \mathrm{mg}$ crosslinked $\mathrm{Na}-\mathrm{HA}$ and $2 \mathrm{mg}$. They found a very minor improvement in the papillary height and attached gingival area which was considered insignificant(24). 
Singh et al 2019 conducted a clinical study to assess the outcome of using injectable HA gels of different concentration $(1 \%, 2 \%, 5 \%)$ for treatment of interdental papillary loss. All the three concentrations were injected into the papilla once a week X 3 weeks. $1 \%$ and $2 \%$ HA did not show any significant improvement. 5\% of $\mathrm{H}$ A group showed $18.2 \%$ papillary heightening at 6 months whereas $1 \%$ HA showed $4.5 \%$ enhancement. Action of $2 \%$ HA cannot be concluded due to inadequate data.(25).

Abdelraouf et al 2019, used $0.1 \mathrm{~mm}$ injectable Restylane (cross-linked HA 20mg/ml) for treatment of black triangles. The gain in height of the papilla in 6 months was $0.06 \pm 0.17 \mathrm{~mm}$ whereas the gain in surface area was found to be $11.8 \pm 30.3$. patients were significantly satisfied with the treatment results as well(26).

Another study was conducted by Mandel et al 2020 where $1 \%$ HA gel was used for the treatment of Class 1 and Class 2 (Norland-Tarnow classification) interdental papillary defects. Group 1 received Flex Barrier, Naturalize $\mathrm{GmbH}$ and Bio Science GmbH, Ransbach Baumbach, Germany which is a combination of non-crosslinked and crosslinked HA gel $\left(2 / 3^{\text {rd }}\right.$ and $1 / 3^{\text {rd }}$ respectively). Group 2 received Revident (CLS LLC, Moscow, Russia) which is similar to the flex barrier with the addition of wheat germ root. Both groups showed a decrease in papillary defects. Both groups showed relapse in 1 week and 1 month although not till the initial level of papilla the results were slightly better in group 2. It was also established that the smaller the defect better is the outcome with both the groups(27).

Turgut and Tamam et al 2020 conducted a clinical trial to compare the interdental papillary fill in maxilla and mandible after injecting HA (hyaDENT BG, BioScience). The HA was filled up to the point where the gingival colour changes to whitish. In 3 months, 1 year and 2 years, the interdental space area left uncovered in maxilla was $0.14,0.09$ and 0.07 (54.21\%, $73.22 \%$, 
$79.35 \%$ coverage) respectively and $0.11,0.07$ and $0.06(57.24 \%, 71.40 \%, 78.71 \%$ coverage $)$ in mandible(28).

\section{ROOT CONDITIONING}

HA was shown to enhance the PDL cell attachment when used for root conditioning as it causes roughening of the dentinal surface.

Babgi et al 2020 used $1 \mathrm{ml}$ of $0.8 \%$ HA gel (Gengigel® $0.8 \%$, Ricerfarma) as a root conditioner on single-rooted teeth and compared the outcomes to 17\% EDTA, 0.2\% Chlorhexidine (CHX). After 48 hours of culture, the teeth treated with HA showed the highest mean value of gingival fibres cell viability $(0.724, \mathrm{p}<0.05)$ whereas CHX, EDTA did not show any improvement when compared to the control group. The authors suggest that HA alters the dentine roughness and hence increases the surface area for the cell attachment. Owing to the antibacterial action of HA the cell viability and growth is also enhanced(29).

\section{INTRABONY DEFECTS}

Pilloni, Nardo and Rojas 2019, treated a 2 walled bone defect caused due to cemental tear using 0.8\% HA (HyaDENT BG, Regedent, BioScience, Dümmer, Germany) and resorbable collagen membrane (Smartbrane, Regedent, BioScience, Dümmer, Germany). After a full-thickness flap reflection, the defect was filled with HA followed by placement of the membrane. 2 years follow up CBCT revealed buccal plate reconstruction and bone fill in the periapical region proving that HA helped in wound healing and bone regeneration(30).

\section{GUIDED TISSUE REGENERATION (GTR)}

HA enhances fibroblast cell adhesion to the cementum and provides an antibacterial environment. Sodium hyaluronate was also shown to have a positive effect on chemical signalling between cells. Hence, it has been widely used as a membrane for GTR(31). 
Tayebi et al 2018 conducted an in vitro study where the efficacy of a combination of materials was evaluated as a GTR membrane. The results suggested that $0.5 \%$ HA in combination with $8 \%$ gelatin and $2 \%$ elastin proved to have an ideal thickness $(150 \mu \mathrm{m})$, biocompatibility, static tensile modules $(1.95 \pm 0.55 \mathrm{MPa})$ and dynamic tensile storage modulus $(314 \pm 50 \mathrm{kPa})$ required for a membrane. The complete separation was accomplished between the epithelial and connective tissues(32).

\section{Peri-implantitis}

Lerma et al 2019 evaluated the efficacy of $0.2 \%$ and $0.8 \%$ HA gel in peri-implantitis patients, enrolled in a randomized control trial, by evaluating the $16 \mathrm{~s}$ ribosomal sequencing for subgingival microorganisms. The study aimed at analysing the antibacterial action of high molecular weight hyaluronic acid (HMW-HA). 0.8\% HA gel was applied in the periimplantitis site in office.The patients were advised to massage the gingiva with the $0.2 \%$ HA gel three times a day for 45 days. The microbiological parameters DNA sequencing were assessed where a significant decrease $(\mathrm{p}<0.05)$ in Streptococcus, Veillonella, Rothia, and Granulicatella, Prevotella, Campylobacter, Anaeroglobulus, Porphyromonas was observed(33).

\section{Nanosystem for drug delivery}

HA has proven to be an ideal drug carrier providing an adequate encapsulation and sustainable release. Turcsanyi et al 2020 conducted a study on various types of chitosan (Chit) modified HA as a nanosized (100-300 nm) drug carriers for vitamin D3 and vitamin E. It was concluded that chit: HA:: 1:1 would be unstable as it is influenced by the acidity and the purity of Chit hence Tripolyphosphate (TCP) was required. TCP treated Chit-HA gave shape stability and encapsulation as a nanoparticle carrier. Chit+TCP+HA shell was used in the 3rd group which 
showed the best results with encapsulation, shape stability, homogeneity and drug carriage for both vitamin D3 and vitamin E. The results of the study proved that HA can be used as a nanodrug delivery system along with Chitosan and TCP as it provides a proper encapsulation and a sustainable drug release system(34).

\section{Newer frontiers}

Research by far has proven HA to be widely advantageous while being biocompatible and non-toxic. Further investigations are required in some fields to identify the true potential of HA. Research is required on the compatibility of HA with various drugs and their forms of delivery. HA has been used for targeted drug delivery in breast cancer(35). The role of HA in dentinal hypersensitivity is not known. Hence, the effects of HA on the exposed dentinal tubules and dentinal hypersensitivity are warranted.

\section{Conclusion}

HA is a vital part of the physiology in humans and other beings. Apart from the naturally occurring hyaluronan, it can be used as a supplement at therapeutic levels for various treatments in different fields. The wide application of HA makes the future perspective of research quite attractive.

\section{REFERENCES:}

1. Amorim S, Reis CA, Reis RL, Pires RA. Extracellular Matrix Mimics Using Hyaluronan-Based Biomaterials. Trends Biotechnol. 2021 Jan;39(1).

2. Dong Q, Guo X, Li L, Yu C, Nie L, Tian W, et al. Understanding hyaluronic acid induced variation of water structure by near-infrared spectroscopy. Sci Rep. 2020 Dec 
$1 ; 10(1)$

3. Aydinyurt HS, Akbal D, Altindal D, Bozoglan A, Ertugrul AS, Demir H. Evaluation of biochemical and clinical effects of hyaluronic acid on non-surgical periodontal treatment: a randomized controlled trial. Irish J Med Sci (1971 -). 2020 Nov 21;189(4).

4. Abatangelo G, Vindigni V, Avruscio G, Pandis L, Brun P. Hyaluronic Acid: Redefining Its Role. Cells. 2020 Jul 21;9(7).

5. Schmidt J, Pilbauerova N, Soukup T, Suchankova-Kleplova T, Suchanek J. Low Molecular Weight Hyaluronic Acid Effect on Dental Pulp Stem Cells In Vitro. Biomolecules. 2020 Dec 28;11(1).

6. IJISRT20OCT463. Int J Innov Sci Res Technol [Internet]. 2020;5(10). Available from: https://ijisrt.com/assets/upload/files/IJISRT20OCT463.pdf

7. Dalessandri D, Zotti F, Laffranchi L, Migliorati M, Isola G, Bonetti S, et al. Treatment of recurrent aphthous stomatitis (RAS; aphthae; canker sores) with a barrier forming mouth rinse or topical gel formulation containing hyaluronic acid: a retrospective clinical study. BMC Oral Health. 2019 Dec 16;19(1).

8. Casale M. Systematic review: the efficacy of topical hyaluronic acid on oral ulcers. Eur PMC. 2017;

9. Pallerla S, Badam R, Boringi M, Pacha V. Hydroxychloroquin: A new hope in the management of oral lichen planus. J Indian Acad Oral Med Radiol. 2015;27(4).

10. Umemura N, Ohkoshi E, Tajima M, Kikuchi H, Katayama T, Sakagami H. Hyaluronan induces odontoblastic differentiation of dental pulp stem cells via CD44. Stem Cell Res Ther. 2016 Dec 20;7(1).

11. Peck MT, Hiss D, stephen L, Olivier A. The in vitro effect of leukocyte- and platelet- 
rich fibrin (L-PRF) and cross-linked hyaluronic acid on fibroblast viability and proliferation. South African Dent J. 2018;73(6).

12. Fujioka-Kobayashi M, Müller H-D, Mueller A, Lussi A, Sculean A, Schmidlin PR, et al. In vitro effects of hyaluronic acid on human periodontal ligament cells. BMC Oral Health. 2017 Dec 16;17(1).

13. Arpağ OF, Damlar I, Altan A, Tatli U, Günay A. To what extent does hyaluronic acid affect healing of xenografts? A histomorphometric study in a rabbit model. J Appl Oral Sci. 2018 Jan 18;26(0).

14. Cirligeriu L, Cimpean A, Calniceanu H, Vladau M, Sarb S, Raica M, et al. Hyaluronic Acid/Bone Substitute Complex Implanted on Chick Embryo Chorioallantoic Membrane Induces Osteoblastic Differentiation and Angiogenesis, but not Inflammation. Int J Mol Sci. 2018 Dec 19;19(12).

15. Hunt DR, Jovanovic SA, Wikesjö UME, Wozney JM, Bernard GW. Hyaluronan Supports Recombinant Human Bone Morphogenetic Protein-2 Induced Bone Reconstruction of Advanced Alveolar Ridge Defects in Dogs. A Pilot Study. J Periodontol. 2001 May;72(5).

16. Zhai P, Peng X, Li B, Liu Y, Sun H, Li X. The application of hyaluronic acid in bone regeneration. Int J Biol Macromol. 2020 May;151.

17. Kim J-J, Song HY, Ben Amara H, Kyung-Rim K, Koo K-T. Hyaluronic Acid Improves Bone Formation in Extraction Sockets With Chronic Pathology: A Pilot Study in Dogs. J Periodontol. 2016 Jul;87(7).

18. Madkour G, EL Refaie I, Mostafa B. ADJUNCTIVE USE OF HYALURONIC ACID WITH SCALING \&amp; ROOT PLANING IN TREATMENT OF CHRONIC 


\section{PERIODONTITIS PATIENTS WITH DIABETES MELLITUS TYPE 2: A} RANDOMIZED CONTROLLED TRIAL. Egypt Dent J. 2018 Oct 1;64(4).

19. Casale M, Moffa A, Vella P, Sabatino L, Capuano F, Salvinelli B, et al. Hyaluronic acid: Perspectives in dentistry. A systematic review. Int J Immunopathol Pharmacol. $2016 \operatorname{Dec} 7 ; 29(4)$.

20. Sadeghi-Ghadi Z, Ebrahimnejad P, Talebpour Amiri F, Nokhodchi A. Improved oral delivery of quercetin with hyaluronic acid containing niosomes as a promising formulation. J Drug Target. 2021 Feb 7;29(2).

21. Pilloni A, Schmidlin PR, Sahrmann P, Sculean A, Rojas MA. Effectiveness of adjunctive hyaluronic acid application in coronally advanced flap in Miller class I single gingival recession sites: a randomized controlled clinical trial. Clin Oral Investig. 2019 Mar 30;23(3).

22. Yıldırım S, Özener HÖ, Doğan B, Kuru B. Effect of Topically-Applied HyaluronicAcid on Pain and Palatal Epithelial Wound Healing: An Examiner-Blind, Randomized, Controlled Clinical Trial. J Periodontol. 2017 Sep 15;

23. Pi S, Choi YJ, Hwang S, Lee D-W, Yook JI, Kim K-H, et al. Local Injection of Hyaluronic Acid Filler Improves Open Gingival Embrasure: Validation Through a Rat Model. J Periodontol. 2017 Nov;88(11).

24. Bertl K, Gotfredsen K, Jensen SS, Bruckmann C, Stavropoulos A. Can hyaluronan injections augment deficient papillae at implant-supported crowns in the anterior maxilla? A randomized controlled clinical trial with 6 months follow-up. Clin Oral Implants Res. 2017 Sep;28(9).

25. Singh S, Vandana K. Use of different concentrations of hyaluronic acid in interdental 
papillary deficiency treatment: A clinical study. J Indian Soc Periodontol. 2019;23(1).

26. Abdelraouf SA, Dahab OA, Elbarbary A, El-Din AM, Zaki BM. Assessment of Hyaluronic Acid Gel Injection in the Reconstruction of Interdental Papilla: A Randomized Clinical Trial. Open Access Maced J Med Sci. 2019 Jun 16;7(11).

27. Mandel I, Farkasdi S, Varga G, Károly Nagy Á. Comparative Evaluation of Two Hyaluronic Acid Gel Products for the Treatment of Interdental Papillary Defects. Acta Stomatol Croat. 2020 Sep 15;54(3).

28. Çankaya ZT, Tamam E. An examination of the 2-year results obtained from hyaluronic acid filler injection for interdental papilla losses. Quintessence Int. 2020;51(4):274-84.

29. Babgi W, Alhajaji M, Al-Mehmadi L, Elbaqli R, Khayat N, Aldahlawi S, et al. Effect of root conditioning agents hyaluronic acid, EDTA and chlorhexidine on the attachment of human gingival fibroblasts to healthy root surface. Saudi Dent J. 2020 Mar;

30. Pilloni A, Nardo F, Rojas MA. Surgical Treatment of a Cemental Tear-Associated Bony Defect Using Hyaluronic Acid and a Resorbable Collagen Membrane: A 2-Year Follow-Up. Clin Adv Periodontics. 2019 Jun 6;9(2).

31. Aveic S, Craveiro RB, Wolf M, Fischer H. Current Trends in In Vitro Modeling to Mimic Cellular Crosstalk in Periodontal Tissue. Adv Healthc Mater. 2021 Jan $16 ; 10(1)$.

32. Tayebi L, Rasoulianboroujeni M, Moharamzadeh K, Almela TK, Cui Z, Ye H. 3DPRINTED MEMBRANE FOR GUIDED TISSUE REGENERATION.

33. Soriano-Lerma A, Magán-Fernández A, Gijón J, Sánchez-Fernández E, Soriano M, García-Salcedo JA, et al. Short-term effects of hyaluronic acid on the subgingival 
microbiome in peri-implantitis: A randomized controlled clinical trial. J Periodontol. 2020 Jun 18;91(6).

34. Turcsányi Á, Varga N, Csapó E. Chitosan-modified hyaluronic acid-based nanosized drug carriers. Int J Biol Macromol. 2020 Apr;148.

35. Li J, Li M, Tian L, Qiu Y, Yu Q, Wang X, et al. Facile strategy by hyaluronic acid functional carbon dot-doxorubicin nanoparticles for CD44 targeted drug delivery and enhanced breast cancer therapy. Int J Pharm. 2020 Mar;578. 